\title{
How Musical Selection Impacts the Performance of the Interaction with the Computer
}

\author{
Mickael da Costa $^{1}$, Davide Carneiro ${ }^{1}$, Marcelo Dias ${ }^{2}$, and Paulo Novais ${ }^{1}$ \\ 1 CCTC/DI, Universidade do Minho, Braga, Portugal \\ dcarneiro@di.uminho.pt, dino,pjon@di.uminho.pt, \\ 2 Escola de Psicologia, Universidade do Minho, Braga, Portugal \\ marcelof vdias@gmail.com
}

\begin{abstract}
In this busy society of ours people push their limits to work better and more in order to remain competitive with their peers. Nonetheless, working longer hours does not necessarily improves productivity nor performance. In order to prevent the negative consequences of this increasing trend, the evolution of performance throughout the day of work should be more closely monitored. This could avoid undesirable states or even breakdowns, which have social and economical implications. In this work we measure user performance through their interaction with the computer. We monitor its evolution during a day of work and how different types of music may increase or decrease its natural daily degradation. We conclude that the relationship between types of music and its effects is not universal and depends, among other things, on the musical profile of the individual. A prototype for a distributed music recommendation service is presented that suggests musics at an individual and group level, based on user musical profiles and objectives.
\end{abstract}

Keywords: Music, Performance, Context Acquisition, Human-Computer Interaction

\section{Introduction}

The Human being is currently under an increasing demand for performance, fruit of a competitive society in which the scarcity of resources drives individuals into harsher conditions. Workplaces are particularly "good" examples of this reality. Lack of jobs, decreasing wages, increasing working hours, working in shifts, competitiveness or unrealistic productivity objectives result in a constant and increasing pressure on the individual.

Numerous studies highlight the negative effects of this lifestyle. [12] show positive mean correlations between overall health symptoms, physiological and psychological health symptoms, and hours of work. [5] analyse the impact of overtime and long work hours on occupational injuries and illnesses, to conclude that these variables depend more on the amount of time worked rather than on the level of risk of the job. In [7], the effects of shift work and extended hours of work are analysed at different levels, including family and social life, performance, fatigue, productivity, health, among others. 
As addressed in detail in [2], there is currently an overwork culture, which is further encouraged by greedy management techniques and job insecurity. While the main objective of management is to increase production, this does not necessarily happen, nor will it increase productivity.

There is thus the need to improve performance or productivity by other means that do not bring along such negative effects. This paper presents such an approach on the problem through the use of music. Indeed, musical selection affects many different aspects of our lives, including our physiology, mood or motivation [1]. The aim of this work is to determine the potential effect of music on the natural degradation of performance that occurs during the workday. Specifically, we want to determine if particular types of music can decrease this natural degradation, contributing to a higher overall performance of the individual. This will be done individually when people can make use of headphones, or in group when only sound systems are available.

\subsection{Music and Its Effects}

Music is one of the oldest forms of cultural expression. Given its effects on the Human being at so many different levels (e.g. emotional, health, physiological), it has been studied in the last decades for many different purposes, including its effects on shopping behavior, its therapeutic possibilities, its effects on sport and exercise and even its effects on our emotions $[6,8,10,13]$.

Among many other areas, music is used in many of nowadays workplaces (e.g. shopping malls, individual stores, surgery rooms, collective transportation). Even if music is not playing openly through installed sound systems, workers are often allowed to work with their personal music using headphones, as happens frequently in software development companies.

The strategies for selecting music are often driven by the objective of activating or calming people. In this work we focus on environments in which groups of people work together, mostly in front of a computer, typically offices (e.g. software development, call center, journals).

\section{Distributed Context Acquisition for Performance Indicators}

To implement the study described in this paper and the developed application prototype, a framework for the acquisition of performance indicators from contextual information was developed. This framework is based on a client-server model 1 . The clients (the computers used by the individuals in the environment) provide information about their users while the server receives it and builds the set of features that feed the decision-making and presentation tier.

The clients use the keyboard and mouse as sensors of user performance. Specifically, the user is monitored through particular Operating System events fired from the use of the computer's mouse and keyboard, as follows: 


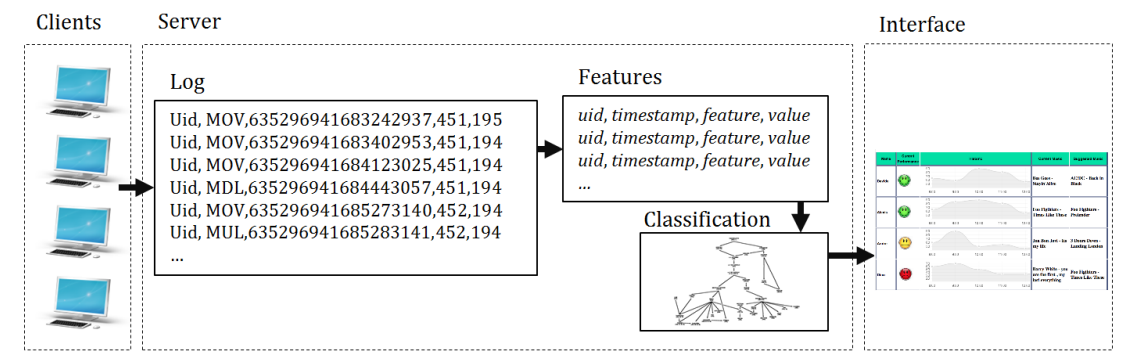

Fig. 1. High-level view of the architecture.

- MOV, timestamp, posX, posY - an event describing the movement of the mouse, in a given time, to coordinates (posX, posY) in the screen;

- MOUSE_DOWN, timestamp, [Left $\mid$ Right], posX, posY - this event describes the first half of a click (when the mouse button is pressed down), in a given time. It also describes which of the buttons was pressed (left or right) and the position of the mouse in that instant;

- MOUSE_UP, timestamp, [Left|Right], posX, posY - an event similar to the previous one but describing the second part of the click, when the mouse button is released;

- MOUSE_WHEEL, timestamp, dif - this event describes a mouse wheel scroll of amount dif, in a given time;

- KEY_DOWN, timestamp, key - identifies a given key from the keyboard being pressed down, at a given time;

- KEY_UP, timestamp, key - describes the release of a given key from the keyboard, in a given time;

The clients share these events in real-time with the server, whom proceeds to compute the following set of features: Key Down Time, Time Between Keys, Mouse Velocity, Mouse Acceleration, Time Between Clicks, Double Click Duration, Average Excess of Distance, Average Distance of the Mouse to the Straight Line, Distance of the Mouse to the Straight Line Between two Clicks, Signed Sum of Angles of the Movement, Absolute Sum of Angles of the Movement and Distance between clicks. These features and the process of their computation are described in more detail in [4].

From these features it is possible to obtain a measure of the user's performance (e.g. an increased distance between clicks or sum of angles represents decreased performance). Then, a wide range of possibilities become real, such as studying the effects of fatigue or stress on performance $[11,4]$ or, as in this case, the effects of musical selection. 


\section{Experimental Study}

In order to determine the influence of music on the interaction of the individuals with their computers and on their behaviour within the environment, an experimental study was carried out. This study aimed to:

- Determine if musical selection (the independent variable) has an effect on the performance of the interaction patterns (the dependent variable) of the users with the computer;

- Determine if different types of music have different effects on the variable;

- Study and quantify the effects of different types of music on the variable;

- Determine if users are conscious of the effects measured or, at least, of some effect at some level;

In the past we have studied how performance is negatively influenced by fatigue throughout the day. In the present study we aim to determine if music may have a positive effect on the performance of the individual by improving it or by delaying its decrease during the day or during particularly stressing periods. The verification of this possibility will support the development of a music recommendation service aimed at improving musical selection with particular objectives, such as improving individual or group performance, satisfaction with music selection or motivation to work. This is expected to consequently improve indicators such as work satisfaction, productivity and quality of the working environment.

\subsection{Method}

This experimental study took place in the Intelligent Systems Lab of the University of Minho. In this lab, numerous students and researchers spend their day working with a specific computer and are allowed to listen to music using headphones. 12 participants were selected to take place in this study, aged between 20 and 28, with an average of 24.3.

Prior to the participation in the study, each individual filled in a questionnaire aimed at determining their musical preferences. Moreover, at the end of each day, they also filled in another questionnaire to determine their subjective opinion about the musical selection of the day.

The selected individuals were requested to participate in the study for five days. During their participation they need not change any of their routines: the

only request was that they carried out their usual tasks while listening to the provided music using their headphones.

The recording of their performance indicators was carried out in the background through a log application that required no interaction at all.

The independent variable in this study was thus musical selection. Five different types of music were used, first classified and put forward by [9] in the form of five so-called mood clusters. Each cluster contained music classified as follows: 
- Cluster 1: passionate, rousing, confident, boisterous, rowdy

- Cluster 2: rollicking, cheerful, fun, sweet, amiable/good natured

- Cluster 3: literate, poignant, wistful, bittersweet, autumnal, brooding

- Cluster 4: humorous, silly, campy, quirky, whimsical, witty, wry

- Cluster 5: aggressive, fiery, tense/anxious, intense, volatile, visceral

The dependant variable was the performance of the participants, measured in terms of the features described previously.

Before the actual start of the data collection, each participant filled in a first questionnaire, meant to establish a profile of each participant. In this questionnaire, each participant provides some standard demographic data, rates some musics from the different clusters according to their level of activation or valence (from the participant's point of view) and answers some questions that allow to perceive their musical preferences.

During the actual study, each participant took part in five different moments of data collection, each one in a different day. In each day, the participant listens to musics from one of the different clusters during the whole period of work, with a minimum of 3 hours.

At the end of each day, each participant answered another questionnaire aimed to determine how the type of music listened made them feel concerning their performance at work (e.g. is the participant consciously aware of some effect?). Moreover, it was also the aim of this questionnaire to determine if the music truly induced the desired state in the participant.

The data collected, from both the questionnaires and the performance monitoring software, was analysed using statistical software and the results are described further ahead in this paper.

\section{$3.2 \quad$ Results}

Given the scope of the paper, we will not dwell to deep into the results of the study: we will only focus on the most important aspects that allow us to grasp the relationship between music and performance.

One of our objectives was to determine if the musical selection in each cluster would be experienced by the participants as expected, i.e., if the clusters we deemed to be calm would be considered calming by the participants. As Figure 2 shows, this happens indeed. Cluster 3, containing music classified as autumnal, brooding or literate, is the one that relaxes participants the most. Cluster 5 , on the other hand, containing music described as aggressive, fiery or tense/anxious, is the one that relaxes them the least.

There is not necessarily a direct relationship between how relaxed you are and your performance. Indeed, this relationship is more complex than it may seem at first sight. In this study we found that our performance does not depend only on the musical selection but also on the musical profile of the individual.

Indeed, if we consider Figure 3, we notice that the Cluster that attenuates fatigue the most over the day is Cluster 4, while the ones that contribute to increasing the effects of fatigue the most are Clusters 2 and 5 . This can be explained 


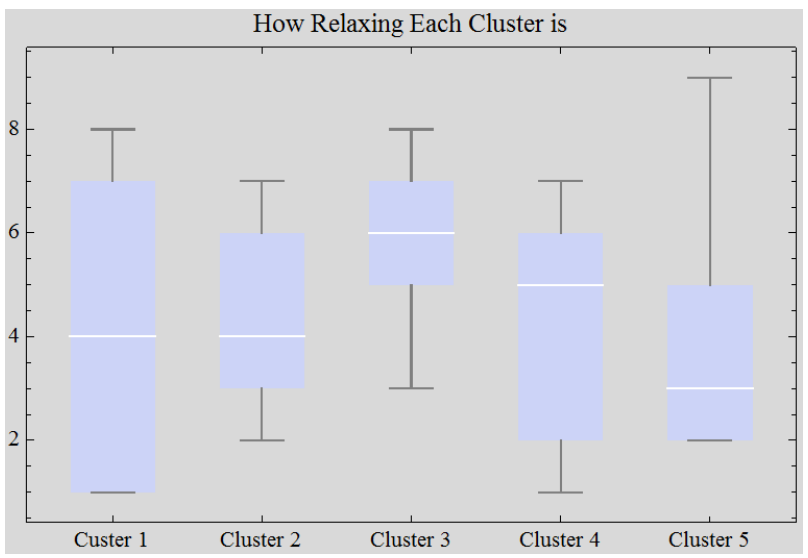

Fig. 2. Distribution of the gradings, by the users, of how relaxing each Cluster is (1 no relaxing at all, 9 - highly relaxing).

by the fact that Cluster 4 contains music that can be described as humorous and silly, contributing to the good mood and motivation of the participant. Cluster 2 , although somewhat similar, is more calm and activates people less. Cluster 5 contains heavy music that, over longer periods, will wear the participants out, producing negative effects. These characteristics can help to understand the observed differences.

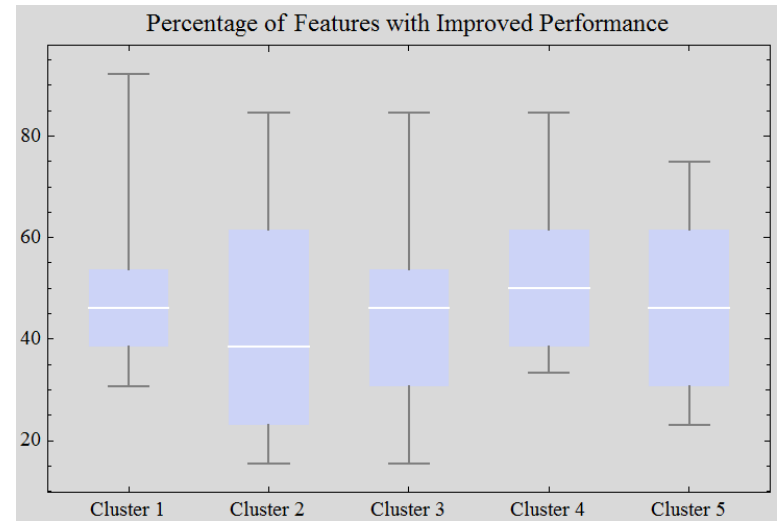

Fig. 3. Distribution of the percentage of features in which each participant improved their performance over the day, for each Cluster.

These differences, although visible when looking at all the population, can result still more interesting when considering individual participants and their musical profile. Indeed, we observe that people that are more into heavy music 
are positively affected and see their performance improved by a longer timespan with the heavier clusters. They are activated by this music in a positive manner and work more efficiently. These are also people that have a higher baseline activation, i.e., they are naturally more "stressed". People that are naturally calmer, on the other hand, find this music annoying and sometimes hurtful to hear and are unable to concentrate, which affects their performance. These individuals thus work with more performance with more calm music.

A good example of this are participants "Davide" and "Vitor Neto" (Figure 4). Davide, who can be described as someone who regularly listens to heavy music, sees achieves the best performance results with Clusters 2 and 5. Vitor, on the other hand, a calmer person by nature, demonstrates better performance in Clusters 3 and 4.

\begin{tabular}{|c|c|c|c|c|c|}
\hline User & Cluster 1 & Cluster 2 & Cluster 3 & Cluster 4 & Cluster 5 \\
\hline Alexis & 53.8462 & 23.0769 & 15.3846 & 38.4615 & 46.1538 \\
\hline Andre & 38.4615 & 46.1538 & 53.8462 & 61.5385 & 69.2308 \\
\hline Angelo & 53.8462 & 23.0769 & 84.6154 & 84.6154 & 61.5385 \\
\hline Claudia & 30.7692 & 23.0769 & 46.1538 & 53.8462 & 46.1538 \\
\hline Davide & 46.1538 & 84.6154 & 46.1538 & 38.4615 & 61.5385 \\
\hline Angelo & 50 & 41.6667 & 33.3333 & 50 & 75 \\
\hline JosePacheco & 61.5385 & 69.2308 & 53.8462 & 38.4615 & 23.0769 \\
\hline Kevin & 41.6667 & 66.6667 & 33.3333 & 33.3333 & 25 \\
\hline Luis & 61.5385 & 30.7692 & 69.2308 & 46.1538 & 61.5385 \\
\hline Ricardo & 92.3077 & 61.5385 & 30.7692 & 61.5385 & 46.1538 \\
\hline VitorNeto & 38.4615 & 38.4615 & 61.5385 & 76.9231 & 30.7692 \\
\hline Dino & 38.4615 & 15.3846 & 30.7692 & 69.2308 & 30.7692 \\
\hline
\end{tabular}

Fig. 4. Percentage of features that improved over the day, for each user and each cluster.

Indeed, the problem of determining the most appropriate style of music for an individual is a complex one and, as these results show, several variables must be taken into account. Namely, and besides the type of music, the musical profile of the individual. Moreover, the objective of the individual at the time (e.g. does he need to complete a task quickly? Does he prefer to work calmly?) as well as the timespan (e.g. we have the tendency to grow tired of a type of music if listen to it for prolonged periods of time) should also be included in the future.

\section{Music Recommendation Service}

Based on the results described in the previous section, we started the development of a prototype for a music recommendation service (Figure 5). This prototype has as main objective to select the most appropriate style of music at a given time. It can do so at two different levels: user-level (in which the prototype optimizes the musical selection for a given user) and group-level (in 
which it does so for a group of people). In both cases, the process is similar. The prototype is a distributed one. Each user is interacting with a particular computer, which is monitoring his performance and client

From the profiles defined through the questionnaires, we know how each individual feels about each type of music: how much they like it, how relaxing/activating they find it or to which extent they preferred to have carried out their activities without listening to this particular type of music.

The users also provide the prototype with their current objective, using a minimalist interface. At any moment, the objective can be to relax (e.g. when the user is involved in a creative task), to activate (e.g. when the user needs to complete a given task quickly) or, aside from performance issues, to listen to their favourite musics. Besides assigning an objective, the user also assigns a weight to Performance and Musical Preference. That is, placing a bigger weight on Performance will result in musics that contribute more to the user's activation, despite his preferences. On the other hand, musics that are more to the taste of the user will be selected, despite less effective results being expected in what concerns performance.

These variables, as well as the weights assigned by the user, are used by optimization functions to attribute a score to each Cluster, at any moment, normalized in the interval [0,1]. To prevent people from getting tired of constantly listening to the same type of music, musics are then selected from all the five Clusters in a frequency that is proportional to these scores (e.g. if Cluster 1 has twice the score of Cluster 2, musics form Cluster 1 will be selected with twice the probability).

A similar process is used for selecting ambient music. However, in this case, it is the ambient manager that determines the objective of the environment. If there is a scheduled brainstorming session, the manager may decide to put activating music in order to stimulate ideas and actions. On the other hand, if the end of the day is approaching, the manager may decide to put more relaxing music as individuals are already tired and activating music may have negative effects.

\section{Conclusion}

Fatigue and its negative effects cause nowadays growing concern. These effects have impact not only at a personal level, wearing one's health, but also at a social level (e.g. our reduced time for social and enjoyable activities) and also at an economical one (companies' costs with absenteeism and reduced productivity are on the rise). Given the current economic scenario, targeting the source of the problem (e.g. decreasing labour time, imposing more favourable legislation) may not be the most realistic or time-efficient solution. In that sense, alternatives should be sought to minimize these negative effects.

In this paper we looked at the possibility of using music to attenuate the negative effects of fatigue on the individual. Specifically, we looked at how per- 


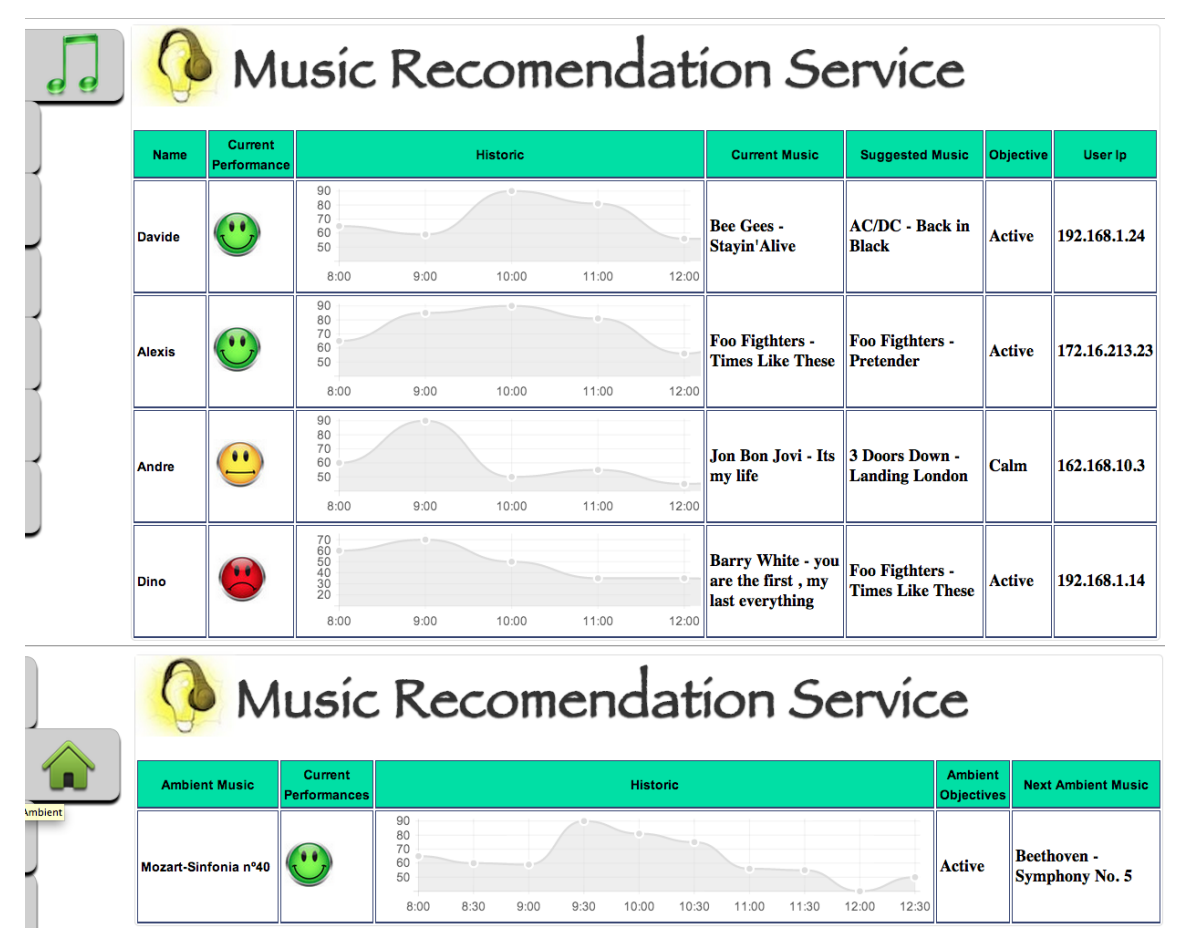

Fig. 5. Detail of the interfaces of the Music Recommendation Service for individual users (upper image) and for the group of users (lower image).

formance, measured in terms of the interaction with the mouse and keyboard, decreases along the day and how different types of music affect this phenomena.

We conclude that the relationship is a complex one and involves variables other than the type of music, including the objective of the individual in each moment and his personal preferences regarding music. The data collected in the experimental study was used to define optimization functions that are used to maximize different aspects of this relationship: to select the favourite musics of the users, to select the music that active the users the most or to select the music that calms them the most.

In future work we will address this problem in more detail, namely by including additional variables that can better shape the relationship between music, performance and fatigue and by analysing different music classification mechanisms.

\section{Acknowledgements}

This work is part-funded by ERDF - European Regional Development Fund through the COMPETE Programme (operational programme for competitiveness) and by National Funds through the FCT - Fundação para a Ciência e a 
Tecnologia (Portuguese Foundation for Science and Technology) within project FCOMP-01-0124-FEDER-028980 (PTDC/EEI-SII/1386/2012).

\section{References}

1. Burns, L., Labbé, E., Arke, B., Capeless, K., Cooksey, B., Steadman, A., Gonzales, C. The effects of different types of music on perceived and physiological measures of stress. Journal of music therapy, 39(2), 101-116 (2002)

2. Bunting, M. Willing Slaves: How the Overwork Culture is Ruling Our Lives. Harper Perennial, ISBN 978-0007163724 (2005)

3. Carneiro, D., Castillo, J. C., Novais, P., Fernández-Caballero, A., Neves, J. (2012). Multimodal behavioral analysis for non-invasive stress detection. Expert Systems with Applications, 39(18), 13376-13389.

4. Carneiro, D. An Agent-based Architecture for Online Dispute Resolution. PhD Thesis. Available at http://repositorium.sdum.uminho.pt/handle/1822/28773 (accessed in April, 2014)

5. Dembe, A. E., Erickson, J. B., Delbos, R. G., Banks, S. M. The impact of overtime and long work hours on occupational injuries and illnesses: new evidence from the United States. Occupational and environmental medicine, 62(9), 588-597 (2005)

6. Gorn, J. The effects of music in advertising on choice behavior: A classical conditioning approach. The Journal of Marketing, 94-101 (1982)

7. Harrington, J. M. Health effects of shift work and extended hours of work. Occupational and Environmental medicine, 58(1), 68-72 (2001)

8. Hatem, P., Lira, I., Mattos, S. The therapeutic effects of music in children following cardiac surgery. Jornal de Pediatria, 82(3), 186-192 (2006)

9. Hu, X., Downie, J. S., Laurier, C., Bay, M., Ehmann, A. F. (2008, September). The 2007 MIREX Audio Mood Classification Task: Lessons Learned. In ISMIR (pp. 462-467).

10. Karageorghis, I., Terry, C. The psychophysical effects of music in sport and exercise: a review. Journal of Sport Behavior, 20(1), 54-68 (1997)

11. Pimenta, A., Carneiro, D., Novais, P., Neves, J. (2013). Monitoring Mental Fatigue through the Analysis of Keyboard and Mouse Interaction Patterns. In Hybrid Artificial Intelligent Systems (pp. 222-231). Springer Berlin Heidelberg.

12. Sparks, K., Cooper, C., Fried, Y., Shirom, A. The effects of hours of work on health: a meta-analytic review. Journal of occupational and organizational psychology, 70(4), 391-408 (1997)

13. Yalch, R., Spangenberg, E. Effects of store music on shopping behavior. Journal of Consumer Marketing, 7(2), 55-63 (1990) 